\title{
Preheating Quantification for Smart Hybrid Heat Pumps Considering Uncertainty
}

\author{
Mingyang Sun, Member, IEEE, Goran Strbac, Member, IEEE, \\ Predrag Djapic, Member, IEEE, Danny Pudjianto, Member, IEEE
}

\begin{abstract}
The deployment of smart hybrid heat pumps (SHHPs) can introduce considerable benefits to electricity systems via smart switching between electricity and gas while minimizing the total heating cost for each individual customer. In particular, the fully-optimized control technology can provide flexible heat that redistributes the heat demand across time for improving the utilization of low-carbon generation and enhancing the overall energy efficiency of the heating system. To this end, accurate quantification of preheating is of great importance to characterize the flexible heat. This paper proposes a novel data-driven preheating quantification method to estimate the capability of heat pump demand shifting and isolate the effect of interventions. Varieties of fine-grained data from a real-world trial are exploited to estimate the baseline heat demand using Bayesian deep learning while jointly considering epistemic and aleatoric uncertainties. A comprehensive range of case studies are carried out to demonstrate the superior performance of the proposed quantification method and then, the estimated demand shift is used as an input into the whole-system model to investigate the system implications and quantify the range of benefits of rolling-out the SHHPs developed by PassivSystems to the future GB electricity systems.
\end{abstract}

Index Terms-Baseline estimation, demand response, deep learning, power system economics, smart hybrid heat pumps.

\section{INTRODUCTION}

D ECARBONIZING the heat sector through electrification will lead to significant increases in peak electricity demand and therefore drive substantial low carbon electricity generation investment and distribution network reinforcement [1]. In this context, the deployment of smart hybrid heat pumps can significantly enhance the capability of the system to integrate and more effectively utilize low-carbon generation, provide balancing services (frequency response and reserve services), reduce low-carbon generation requirements, and eliminate investment in additional generating capacity needed for maintaining system security and distribution network reinforcement [2].

Conventionally, hybrid heat pump (HHP) is controlled either on a time-switch, which may result in an inefficiently high temperature of heating water, or on a constant weathercompensated heating water temperature, which renders redundant heat loss during the midnight [3]. To address these issues, a fully-optimized control strategy with predictive demandside management and proactive consumer engagement was developed in the Freedom project [4] based on Predictive

M. Sun, G. Strbac, P. Djapic and D. Pudjianto are with the Department of Electrical \& Electronic Engineering, Imperial College London, London, SW7 $2 \mathrm{AZ}, \mathrm{UK}$
Demand Control (PDC) technology that aims to optimize the performance of the heating system over the upcoming day and minimize energy consumption and energy cost while satisfying the comfort requirement for each individual customer. More specifically, smart control allows the heat pump to run gently using PDC and to gradually ramp up the heat pump overnight via a dynamically controlled flow temperature. Additionally, the smart control technology enables the HHP to learn the thermal characteristics of the property and then build a specific model for this property and heat system. For traditional HHP, control is conducted by switching between electricity and gas only based on external temperature. However, for the smartly controlled heating systems, the dynamic control approach enables it possible to make COP influenced by the heating water temperature as much as the external temperature. Based on building thermal inertia, smart control provides the benefits of enhancing demand flexibility and reducing the total bill for customers by self-diagnosing cost-efficient demand reduction measures that could be applied to the home to increase fabric storage. More information regarding the considered smart control technology can be found in [5].

As one of the critical benefits, flexible heating provided by such fully-optimized control technology is based on preheating that involves heating the households earlier than would be done otherwise while utilizing inherent heat storage in the fabric of the houses. The volume of demand shifted through preheating is used as a proxy to quantify the flexibility of shifting HHP demand in time in response to price signals. The flexibility of SHHPs based on switching between electricity and gas can be used to reduce peak demand and the corresponding investment in additional generation and network reinforcement. Preheating is also a strategy that redistributes heat demand across time in order to improve utilization of lowcarbon generation and enhance the overall energy efficiency of the heating system. In particular, preheating is fundamentally driven by the external temperature and the ratio of gas and electricity prices, denoted by the gas cost ratio (GCR); a higher GCR value will activate the electric heat pump to supply the heat demand, whereas a lower GCR value (i.e., higher electricity price) will enable the heat demand supplied by the gas boiler. Given the above preheating could give the characteristics of fabric of building and perhaps preheating could be estimated for other conditions i.e. external temperatures. This could be then used, for example for, analysis of benefit of preheating if conventional HP are installed as well.

To accurately quantify the preheating of SHHPs for residential customers, baseline heat demand estimation is a critical 
procedure to estimate the heat consumption under normal price conditions (e.g., flat tariff) during the event periods (e.g., Economy 7 tariff or Economy 10 tariff) in the context that the highest room temperature set point requested by the user in this period is relatively lower. Then the difference between the actual measured heat demand supplied by electric heat pump and the estimated baseline heat demand represents the preheating that can contribute to reducing the total heating cost based on the optimized control technology. Overestimation of baseline heat demand will result in lower heat demand flexibility considered in system operation, which may increase the operation cost. On the other hand, underestimating the baseline will overestimate the flexible heat and thus may lead to considerable impact on system reliability and stability.

In the literature, a number of baseline estimation methods have been proposed and can be generally categorized into similar-day approaches, morning-adjustment approaches, regression approaches, and CONTROL group approaches [6], [7]. First, similar-day methods aim to select the most similar days prior to the event day based on the weather conditions and then averages them to construct the final estimated baseline demand (e.g., LowXofY [8], HighXofY [9]), which may result in tremendous estimation error due to neglecting information of the event day (demand response event). To this end, morning-adjustment methods are proposed by using the actual measured pre-event load data to adjust the estimated baseline demand for further enhancing the estimation performance [10]. Nevertheless, the difference between the actual and estimated load during the morning periods utilized for the adjustment may be due to the effect of demand shift for a single customer, which may mislead the estimation result during the event periods.

Using a regression model to "forecast" the baseline load pattern during the event periods can account for the day information as input features and the target is to establish a nonlinear function to approximate the complex relationship between the selected features and the baseline demand (e.g., [9]). However, demand response events are usually designed for the specific days with extreme weather conditions, thus resulting in the issue of insufficient historical measurements required to build a reliable regression model. To overcome the aforementioned problems existing in conventional methods, synchronous information from the customers who did not participate in the demand response (DR) trial, but who have exhibit similar load patterns to the participants, can be exploited to enhance the estimation performance and tackle the challenges of limited data during the extreme days. This type of approach and the corresponding non-DR customers are known as the the CONTROL group method and the CONTROL group, respectively. For example, the authors in [11] designed a novel CONTROL group baseline estimation method at the household level by selecting the optimal CONTROL group for each DR customer. Although superior performance of the CONTROL group methods have been demonstrated, there still exist a series of issues: 1) selecting the optimal CONTROL group based on the averaged daily load profiles of each cluster cannot effectively handle the customers with distinctive electricity consumption behaviors;
2) the optimal match between the DR and non-DR customers may change across different weather conditions. As one of the effective solutions, a synchronous pattern matching (SPM)based estimated approach is proposed in [6] without requiring historical data and selects the optimal match for each event day of each DR customer.

Although a series of data-driven approaches have been developed for baseline estimation, most of them cannot represent uncertainty and focus on point estimation, which can only provide a single value for each time step during the event hours. With the increasing penetration of renewable energy resources and the volatile energy consumption behavior, massive uncertainties injected from different resources need to be wellcaptured while performing the estimation. Therefore, recent work has tended toward generating a distribution over a certain interval to represent the uncertainty via performing probabilistic estimation. In [12] and [13], Gaussian Process regression and quantile regression are employed as key techniques to generate the probabilistic estimates, respectively. Nevertheless, it is notable that all the above-mentioned existing approaches have been designed for estimating baseline electricity demand whereas we need to investigate and develop a new method for baseline heat demand estimation.

In recent years, neural networks, especially deep neural networks, have attracted considerable attention in the field of energy forecasting, including electricity load forecasting (e.g., [14], [15]) and renewable energy forecasting (e.g., [16], [17]). Although dominant forecasting accuracy can be obtained via deep learning, conventional deep neural networks fail to represent uncertainty, which is the fundamental requirement for probabilistic estimation in this work. To enable deep learning to represent uncertainty, a new research direction, Bayesian deep learning [18], has been investigated to make deep learning more explainable with the advantages of understanding generalization, reliable prediction, and uncertainty representation.

To this end, based on Bayesian deep learning, a novel probabilistic preheating quantification framework is proposed in this paper for estimating the flexible heating provided by SHHPs. According to the available measurements collected from a real SHHP trial, a composite scheme is designed, consisting of a feature construction step to select the most correlated explanatory variables, an estimation model training step to build the Bayesian deep neural network, and a sampling step to finally generate the probabilistic estimates based on the test data. Numerical case studies are carried out to demonstrate that the proposed framework outperforms existing methods. Note that since very limited work has been conducted for preheating quantification, we implement electricity baseline demand estimation methods for comparison in this case. Furthermore, the estimated heat demand shift is employed as an input into the whole-system modeling to investigate the benefits of SHHPs developed by PassivSystems to future GB electricity systems. Note that the proposed quantification approach is a general framework that can also be exploited for other types of HHPs with flexible heating. The key contributions of this paper can be summarized as follows:

(1) A novel preheating quantification framework is pro- 
posed to estimate the heat demand flexibility provided by the smart control technology of hybrid heat pumps with the consideration of uncertainty using the proposed Bayesian deep bi-directional LSTM network. To the best of the authors' knowledge, this is the first work to combine probabilistic graphical models (PGM) with deep bidirectional LSTM networks to simultaneously capture the model and inherently stochastic uncertainties as well as the forward and backward dependencies for baseline heat demand estimation.

(2) This research is based on real fine-grained data collected from the world's first sizable field trial of smart hybrid heat pumps with full demand flexibility. In particular, a fullyoptimized control technology is developed by PassivSystems in the trial with predictive demand-side management and proactive consumer engagement.

(3) The potential benefits of rolling-out SHHPs to the future GB electricity systems are investigated and quantified, for the first time, by inputting the estimated heat demand flexibility into the whole-system model.

The rest of this paper is organized as follows. Section II presents the key challenges of preheating quantification. Section III details the proposed preheating quantification framework. Section IV introduces the proposed Bayesian deep bi-LSTM network. Section IV presents case studies demonstrating the value of the proposed approach. Finally, Section IV discusses conclusions and future extensions of this work.

\section{Key Challenges of Preheating Quantification}

In general, energy flexibility can be characterized from the aspects of total demand increase or decrease, energy demand change duration, maximum power change in demand and response time [19]. In this paper, heat demand flexibility provided by the preheating strategy, which utilizes inherent heat storage in the fabric of the houses to heat the household earlier than the customer request, can effectively shift the demand to avoid peak periods, bringing about tremendous benefits to future electricity systems. Nevertheless, how to accurately quantify preheating considering uncertainty is still an open question with the following main challenges:

Challenge \#1 - Data Availability: In recent years, although researchers have begun to investigate the utilization and contributions of preheating, most of existing work focuses either on electric heat pumps, which can reduce the carbon footprint of heat sector but may lead to significant increases in peak electricity demand, or developing model-based approaches to consider preheating in the system operation and planning model, which cannot explicitly depict the heat demand flexibility. To this end, data-driven preheating quantification of smart hybrid heat pumps is an imperative and crucial research topic, requiring a massive amount of diversified fine-grained data such as customer highest temperature set point, external temperature, electricity price, gas price, heat provided by a gas boiler and heat pump. As one of the most comprehensive studies of HHPs, the world's first sizable field trial of fully optimized hybrid heat pumps with full demand flexibility was carried out in the FREEDOM project and provides a valuable opportunity to identify the "real" heat demand flexibility based on high-resolution measurements [4].
Challenge \#2 - Model and Inherently Stochastic Uncertainties: First, model uncertainty is defined as the uncertainty in the parameters and structure of the considered estimation model, indicating to what extent the model is uncertain or certain in terms of its estimates. In particular, while performing probabilistic baseline heat demand estimation, the established model should be capable of describing how confident the model is to achieve accurate estimates across various weather conditions and tariff types. On the other hand, inherently stochastic uncertainty arises directly from the heat demand data, injected from gas and electricity prices, climate factors, renewable power, and human activities. Additionally, stochastic uncertainty refers to the noise within the data caused by measurement imprecision and thus cannot be explained even with more observations. Furthermore, stochastic uncertainty refers to aleatoric uncertainty, which can be classified into two categories, namely, homoscedastic uncertainty and heteroscedastic uncertainty [20]. The former is task-dependent uncertainty, which remains constant for all input data, and the latter indicates the input-dependent uncertainty. To quantify the preheating of smart hybrid heat pumps, it is imperative but challenging to simultaneously consider both the model and inherently stochastic uncertainties in a single probabilistic estimation model.

Challenge \#3 - Forward and Backward Dependencies: Baseline heat demand estimation, which is the core step of the proposed preheating quantification framework, is generally conducted after the trial (i.e., post-experiment analysis). Therefore, unlike conventional heat load forecasting problem, which can only utilize historical data, one of the characteristics of preheating quantification is that both the pre-event data (i.e., "historical" data) and post-event data (i.e., "future" data) can be jointly considered to estimate the baseline data between them. To this end, capturing forward and backward dependencies in the time series heat data can fundamentally enhance the estimation performance, which will result in a more accurate quantification of heat demand flexibility of SHHPs and thus render a reliable benefit and implication analysis result for future electricity systems.

\section{Proposed Bayesian DeEp LeARning-BASED PREHEATING QUANTIFICATION FRAMEWORK}

To address the aforementioned challenges, a novel flexibility quantification framework is proposed to estimate the heat demand shift attributed to smart hybrid heat pumps at the household level with the consideration of uncertainties. As shown in Fig. 1, the overall workflow of the flexibility quantification framework consists of four main steps including Data Preprocessing, Feature Construction, Bayesian Bidirectional Deep LSTM Neural Network Training, and Preheating Quantification. Detailed illustrations and rationales of the design for each step will be given in the following parts.

\section{A. Step 1. Data Preprocessing}

Before introducing the data preprocessing procedure, brief descriptions about various types of measurements during the 


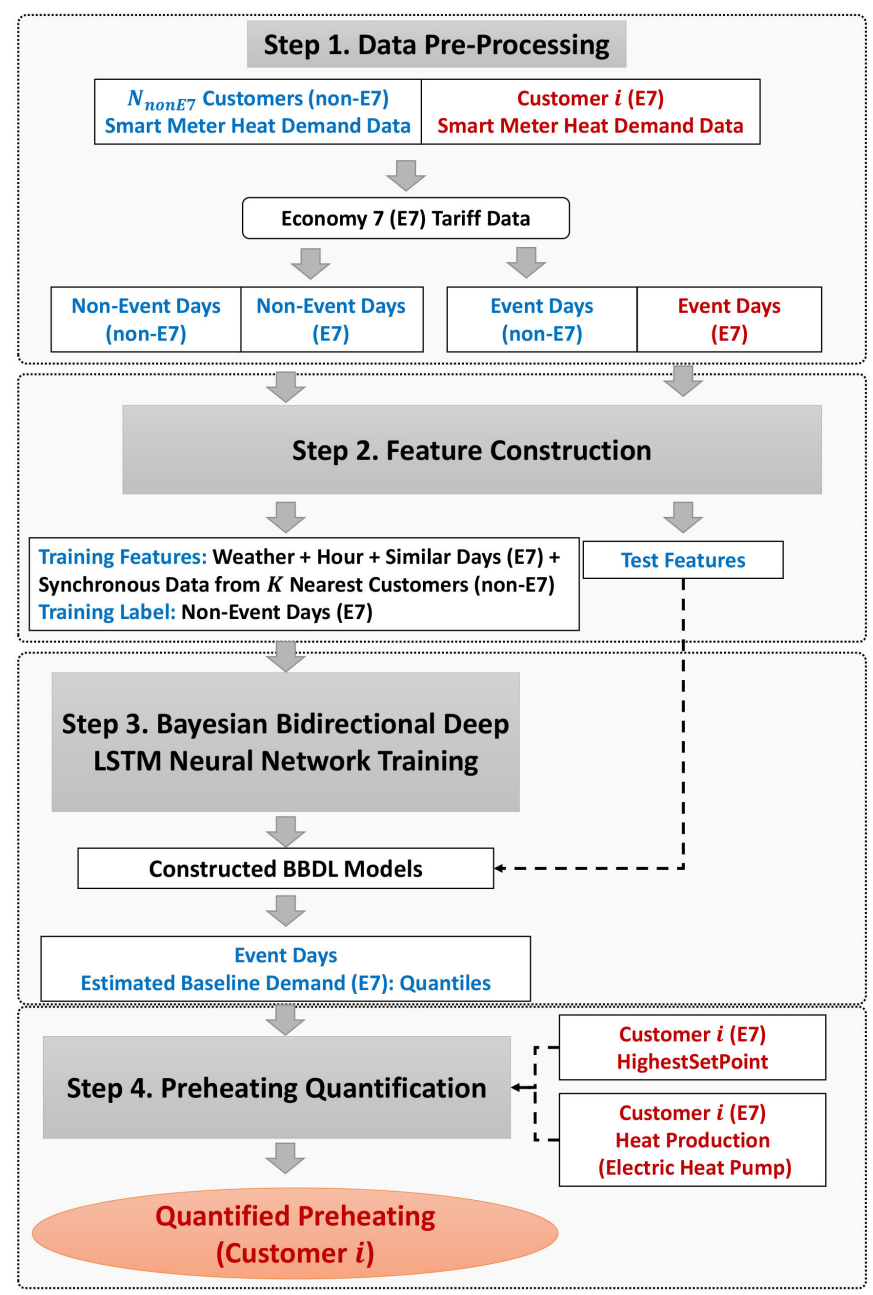

Fig. 1. Overall workflow of the proposed flexibility quantification method (an example of a customer with the Economy 7 electricity tariff).

trial for flexibility quantification are given for each household as follows:

- $H_{E}$ : Heat produced by the heat pump in $\mathrm{kW}$, only including times when there was no request for heat from the gas boiler;

- $H_{G}$ : Heat output from the gas boiler in $\mathrm{kW}$;

- $D$ : Heat demand in $\mathrm{kW}$ calculated by summing the heat outputs of $H_{E}$ and $H_{G}$ (i.e., heat demand should be equal to the total heat production);

- $\bar{T}^{\text {set }}$ : Highest room temperature set point requested by the user;

- $T^{e x t}$ : External temperature measured from the nearest MetOffice weather station;

- П: Tariff type 0-flat tariff, 1-Economy 7 tariff (00:3007:30 70\% of base price, other times 107\% of base price), 2-Economy 10 tariff (0:00-05:00, 13:00-16:00, 20:0022:00 70\% of base price, other times 107\% of base price).

Given the whole dataset for all the participating customers, the first step is to clean the raw data via imputing the missing data and handling the outliers. In this case, we simply impute the missing data and replace the outliers with the average value of the corresponding column. Afterwards, any customers with fewer than a certain proportion of total measurements (e.g., 50\%) are excluded. Then all the customers are classified into three categories: the E7 group, the E10 group, and the Flat group. In this case, as shown in Fig. 1, we illustrate the proposed methodology based on the assumption that the test customer is from the E7 group and all the other customers belonging to E10 and Flat groups are combined to the non-E7 group. Let $N$ denote the total number of customers participating in the trial, the dataset of heat demand for all the customers $D=\left[d_{1}, \ldots, d_{N}\right] \in \mathbb{R}^{T \times N}$ can be separated to $D^{\text {nonE7 }} \in \mathbb{R}^{T \times N_{\text {nonE7 }}}$ for the non-E7 group and $D^{E 7} \in \mathbb{R}^{T \times N_{E 7}}$ for the $E 7$ group, where $N_{n o n E 7}$ and $N_{E 7}$ represent the number of customers in the non-E7 group and the E7 group, respectively.

Furthermore, assuming that the target is to quantify the flexibility of SHHP for customer $i$ from the E7 group, according to the tariff data of customer $i$, defined as $\Pi_{i}$, both $D^{n o n E 7}$ and $d_{i}^{E 7}$ can be further partitioned into heat demand during E7 event days $D^{\text {nonE7, event }} \in \mathbb{R}^{T_{\text {event }} \times N_{\text {nonE7 }} \text {, }}$ $d_{i}^{E 7, \text { event }} \in \mathbb{R}^{T_{\text {event }} \times 1}$ and heat demand during non-event days $D^{\text {nonE7,nonEvent }} \in \mathbb{R}^{T_{\text {nonEvent }} \times N_{\text {nonE }} 7}, d_{i}^{\text {E7,nonEvent }} \in$ $\mathbb{R}^{T_{\text {nonEvent }} \times 1}$, respectively. In addition, all the other parameters are separated into the corresponding groups according to the tariff data as described above.

\section{B. Step 2. Feature Construction}

Based on the above separated groups of data, the main target in this step is to establish the training and test sets for constructing the probabilistic estimation model to handle the challenges illustrated in Section II. First, for Challenge \# 3, the input features should contain the information before and after the events. Thus, given the target estimating time step $t$, the key selected training features consist of F1) the heat demand historical data of customer $i$ at the same time step on the previous day, denoted by $d_{i, t-24}^{E 7, \text { nonEvent }}$; F2) $d_{i, t-25}^{E 7, \text { nonEvent }}$; F3) the heat demand historical data of customer $i$ at the same time step on the previous two days, denoted by $\left.d_{i, t-48}^{E 7, \text { nonEvent }} ; \mathrm{F} 4\right)$ $d_{i, t-49}^{E 7, \text { novent }}$; F5) the heat demand future data of customer $i$ at the same time step on the next day, denoted by $d_{i, t+24}^{E 7, n o n E v e n t}$; F6) $d_{i, t+25}^{E 7, \text { novent }}$; F7) the heat demand future data of customer $i$ at the same time step on the next two days, denoted by $d_{i, t+48}^{E 7, \text { nonent }}$; F8) $d_{i, t+49}^{E 7, \text { nonEvent }}$.

To fully utilize the synchronous data from similar nonE7 customers, an additional procedure is to select the most correlated customers, also referred to as nearest neighbors, based on appropriate distance metrics. Let $K$ denote the number of nearest neighbors that we would like to select from the non-E7 group for the tested customer $i$, the first step is to calculate the Pearson product-moment correlation coefficient $r_{i, j}$ between each pair of $d_{i}^{E 7, \text { nonEvent }}$ and $d_{j}^{\text {nonE } 7, \text { nonEvent }}$ for all $j=1, \ldots, N_{n o n E 7}$, following the equation below:

$$
r_{i, j}=\frac{\operatorname{cov}\left(d_{i}^{E 7, \text { nonEvent }}, d_{j}^{\text {nonE7,nonEvent }}\right)}{\sigma_{i} \sigma_{j}}
$$

where $\operatorname{cov}=E\left[\left(d_{i}^{E 7, \text { nonEvent }}-\mu_{i}\right)\left(d_{j}^{\text {nonE } 7, \text { nonEvent }}-\mu_{j}\right)\right]$ is the covariance, $\mu$ is the mean value of the vector, $\sigma$ represents 
the standard deviation. Then the set of the $K$ most correlated customers $J^{*}=\left[j_{1}^{*}, \ldots, j_{K}^{*}\right]$ can be identified by selecting the $j_{k}^{*}$ s that corresponds to the $k$ th highest absolute value of $r_{i, j}$. In this way, beyond the above selected eight types of features, additional features extracted from the non-E7 group are the synchronous data F9) $\left\{d_{j_{k}^{*}, t}^{\text {nonE7,nonEvent }}\right\}_{k=1}^{K}$. It is notable that, if the examined E7 customer exhibits abnormal heat consumption behavior, it will be ineffective to use the identified "nearest" nonE7 customers as features and the estimation accuracy may get reduced. To this end, a correlation limit (e.g., $\left|r^{m i n}\right|=0.2$ ) can be determined and thus, no features of the nonE7 customers will be included if the absolute value of correlation between the identified most correlated nonE7 customer and the examined E7 customer is lower than this limit.

Furthermore, one of the similar-day methods, High5of10, is performed to construct the features that leverage the information from the tested E7 customer itself. This can be achieved by using the average load of five days in the past ten non-event days prior to the tested event day, denoted by F10) $d_{t}^{E 7, A v e}$. Finally, the corresponding external temperature data F11) $T_{t, i}^{e x t}$, the highest room temperature set point F12) $T_{t, i}^{s e t}$, and the hour of the day F13) $h_{t}$ are included as additional features to consider the impacts of climate factor and human behavior, respectively. To summarize, the constructed explanatory variables $X^{\text {train }}$ can be expressed as follows:

$$
\begin{gathered}
X^{\text {train }}=\left[d_{i, t-24}^{E 7, \text { nonEvent }}, d_{i, t-25}^{E 7, \text { nonEvent }}, d_{i, t-48}^{E 7, \text { nonEvent }},\right. \\
d_{i, t-49}^{E 7, \text { nonEvent }}, d_{i, t+24}^{E 7, \text { nonEvent }}, d_{i, t+25}^{E 7, \text { nonEvent }}, d_{i, t, \text { nonEvent }}^{\text {E7 }}, \\
d_{i, t+49}^{E 7, \text { nonEvent }}, d_{j_{1}^{*}, t}^{\text {nonE } 7, \text { nonEvent }}, \ldots, d_{j_{K}^{*}, t}^{\text {non } E 7, \text { nonEvent }}, \\
\left.d_{t}^{E 7, \text { Ave }}, T_{t, i}^{\text {ext } t}, T_{t, i}^{\text {set }}, h_{t}\right]
\end{gathered}
$$

The same procedure is carried out to construct the test dataset $X^{\text {test }}$ based on $D^{\text {nonE7,event }} \in \mathbb{R}^{T_{\text {event }} \times N_{\text {nonE7 }} \text { and }}$ $d_{i}^{E 7, \text { event }} \in \mathbb{R}^{T_{\text {event }} \times 1}$ during the $\mathrm{E} 7$ event days. The training label is represented by:

$$
y^{\text {train }}=d_{i}^{E 7, \text { nonEvent }}
$$

If we consider a non-E7 customer as the tested customer for evaluating the estimation performance, the test label can be regarded as the real baseline heat demand during the E7 event periods, denoted by $y^{\text {train }}=d_{i}^{E 7, \text { Event }}$.

\section{Step 3. Bayesian Deep Bi-LSTM Neural Network Training}

This step is the core procedure of the proposed framework that deals with the challenge of capturing model and stochastic uncertainties (Challenge \#2) via combining Bayesian network with deep learning and considering both forward and backward dependencies (Challenge \# 3) via employing the bidirectional architecture of a recurrent neural network. Detailed explanations of the proposed Bayesian deep Bi-LSTM neural network (BDBL) will be given in the next section.

For the tested E7 customer $i$, the BDBL network $B D B L_{i}$ will be trained based on the established input features $X^{\text {train }}$ and the true label $y^{\text {train }}$. Hypeparameters of $B D B L_{i}$ (e.g., number of layers, number of neurons in each layer, learning rate, dropout) need to be tuned through grid search and crossvalidation in this step. After building the estimation model $B D B L_{i}$, given the test features $X^{\text {test }}$, the estimated baseline heat demand during the E7 event periods $\hat{y}^{\text {test }}$ is the output of this step. Note that $\hat{y}^{\text {test }}$ consists of $N_{\text {sample }}$ samples for each time step $t$ generated from the proposed BDBL network, which can represent the massive uncertainties injected from different resources.

\section{Step 4. Preheating Quantification}

The flexibility of SHHPs provided to the electricity system can be quantified by estimating how much of the heat pump demand has been shifted compared to the baseline heat demand based on a flat electricity tariff. It is imperative to note that not all time steps during the low-price windows in the E7 experiments are automatically classified as involving preheating. In other words, the preheating of homes using cheaper electricity (mostly overnight) is evaluated as proxy for the flexibility of shifting hybrid heat pump demand in time in response to the price signals. The preheating periods for a given customer should be detected based on the customers' highest room temperature set point so that the preheating intervals only include those periods of low-price windows that have lower temperature set points compared to the highest set point value over a day.

Mathematically, given the heat output data produced by the electric heat pump during the event hours, denoted by $H_{G}^{E 7, \text { event }}$, and the corresponding highest temperature set point $\bar{T}^{\text {set,event }}$, the quantified preheating can be calculated as:

$H_{P}(:, t)= \begin{cases}H_{G}^{E 7, \text { event }}(t)-\hat{y}^{\text {test }}(:, t) & \text { if } \bar{T}_{t}^{\text {set, event }}<\bar{T}^{\text {high }} \\ 0 & \text { otherwise }\end{cases}$

where $H_{P}(:, t)$ represents the probabilistic preheating estimates at time step $t$ and $\bar{T}^{\text {high }}$ is the user-defined relatively higher set point value over a day.

\section{Proposed Bayesian Deep Bi-LSTM Network}

Deep learning has obtained prominent achievements in various challenging areas of artificial intelligence, consisting of "hearing" (i.e., speech recognition), "seeing" (i.e., image recognition), and "reading" (i.e., natural language processing) [21], [22]. Regarding the time-series forecasting tasks in energy systems, recurrent neural nets (RNNs) exhibit superior performance over other types of neural nets (e.g., CNN) via mapping the historical inputs to each output; however, they also suffer from the vanishing gradient problem [23]. To tackle this problem, a special RNN architecture, long short-term memory (LSTM), is proposed in [24] that can deal with long-term dependencies in sequential data. More specifically, an LSTM cell is composed of an input gate, a forget gate, an output gate, and an internal state to realize the functionality of preserving and retrieving information over a long time period. As introduced in Section II, the identified challenge (i.e., Challenge \#3) for flexibility quantification is 
to estimate the baseline heat demand during the event periods with the consideration of both pre-event and post-event data. In other words, the estimation model should be capable of simultaneously capturing forward and backward dependencies to boost the performance.

To this end, a more sophisticated structure of LSTM, bidirectional LSTM [25], is a natural fit for baseline heat demand estimation to deal with Challenge \#3. Fig. 2 presents the unfolded topological graph of a deep bidirectional LSTM network (DBLSTM) with $N_{L}$ hidden layers, aiming to extract the high-level representation of baseline heat demand considering both historical and future information and thus enhancing the estimation accuracy. Given the input features $X=\left\{x_{t}\right\}_{t=1}^{T} \in$ $\mathbb{R}^{T \times M}$ and the target output $y=\left\{y_{t}\right\}_{t=1}^{T} \in \mathbb{R}^{T}$, the hidden state and the memory cell state of hidden layer $l$ at time step $t$ are denoted by $h_{l}^{t}$ and $c_{l}^{t}$, respectively. Then the bidirectional LSTM structure of the $l$ th hidden layer can be expressed as

$$
\begin{aligned}
\vec{f}_{t}^{l} & =\sigma\left(\vec{W}_{\vec{h}^{l-1} \vec{h}^{l}} \vec{h}_{t}^{l-1}+\vec{W}_{\vec{h}^{l}}^{f} \vec{h}^{l} \vec{h}_{t-1}^{l}+\vec{b}_{l}^{f}\right) \\
\vec{i}_{t}^{l} & =\sigma\left(\vec{W}_{\vec{h}^{l-1} \vec{h}^{l}}^{i} \vec{h}_{t}^{l-1}+\vec{W}_{\vec{h}^{l}}^{i} \vec{h}_{h}^{l} \vec{h}_{t-1}^{l}+\vec{b}_{l}^{i}\right) \\
\vec{o}_{t}^{l} & =\sigma\left(\vec{W}_{\vec{h}^{l-1} \vec{h}^{l}}^{o} \vec{h}_{t}^{l-1}+\vec{W}_{\vec{h}^{l}}^{o} \vec{h}^{l} \vec{h}_{t-1}^{l}+\vec{b}_{l}^{o}\right) \\
\vec{g}_{t}^{l} & =\tanh \left(\vec{W}_{\vec{h}^{l-1}}^{g} \vec{h}^{l} \vec{h}_{t}^{l-1}+\vec{W}_{\vec{h}^{l}}^{g} \vec{h}^{l} \vec{h}_{t-1}^{l}+\vec{b}_{l}^{g}\right) \\
\vec{c}_{t}^{l} & =\vec{f}_{t}^{l} \odot \vec{c}_{t-1}^{l}+\vec{i}_{t}^{l} \odot \vec{g}_{t}^{l} \\
\vec{h}_{t}^{l} & =\vec{o}_{t}^{l} \odot \tanh \left(\vec{c}_{t}^{l}\right)
\end{aligned}
$$

$$
\begin{aligned}
& \overleftarrow{f}_{t}^{l}=\sigma\left(\overleftarrow{W}_{\overleftarrow{h}^{l}-1 \overleftarrow{h} l}^{f} \overleftarrow{h}_{t}^{l-1}+\overleftarrow{W}_{\overleftarrow{h} l}^{f} \overleftarrow{h}_{h} \overleftarrow{h}_{t-1}^{l}+\overleftarrow{b}_{l}^{f}\right) \\
& \overleftarrow{i}_{t}^{l}=\sigma\left(\overleftarrow{W}_{\overleftarrow{h}^{l-1}}^{i} \overleftarrow{h}_{l} \overleftarrow{h}_{t}^{l-1}+\overleftarrow{W}_{\overleftarrow{h} l}^{i} \overleftarrow{h}^{l} \overleftarrow{h}_{t-1}^{l}+\overleftarrow{b}_{l}^{i}\right) \\
& \overleftarrow{o}_{t}^{l}=\sigma\left(\overleftarrow{W}_{\overleftarrow{h}^{l}-1}^{o} \overleftarrow{h}^{l} \overleftarrow{h}_{t}^{l-1}+\overleftarrow{W}_{\vec{h}^{l}}^{o} \overleftarrow{h}^{l} \overleftarrow{h}_{t-1}^{l}+\overleftarrow{b}_{l}^{o}\right) \\
& \overleftarrow{g}_{t}^{l}=\tanh \left(\overleftarrow{W}_{\overleftarrow{h}^{l-1} \overleftarrow{h}^{l}}^{g} \overleftarrow{h}_{t}^{l-1}+\overleftarrow{W}_{\overleftarrow{h}^{l} l \overleftarrow{h}^{l}}^{g} \overleftarrow{h}_{t-1}^{l}+\overleftarrow{b}_{l}^{g}\right) \\
& \overleftarrow{c}_{t}^{l}=\overleftarrow{f}_{t}^{l} \bigodot \overleftarrow{c}_{t-1}^{l}+\overleftarrow{i}_{t}^{l} \bigodot \overleftarrow{g}_{t}^{l} \\
& \overleftarrow{h}_{t}^{l}=\overleftarrow{o}_{t}^{l} \bigodot \tanh \left(\overleftarrow{c}_{t}^{l}\right)
\end{aligned}
$$

where $i, o, f$, and $c$ represent the input gate, output gate, forget gate, and cell activation vectors, respectively, $\sigma$ is the logistic sigmoid function, $\odot$ is the element-wise product, $W$ represents the weight matrix, and $b$ is the bias. In addition, the symbols $\rightarrow$ and $\leftarrow$ indicate the forward and backward processes, respectively. Then the final representation in the hidden layer $l$ at time step $t$ can be obtained by concatenating the forward and backward state, represented by $h_{t}^{l}=\vec{h}_{t}^{l} \oplus \overleftarrow{h}_{t}^{l}$ if $l>0$, otherwise $h_{t}^{0}=x_{t}$. As seen, the bidirectional LSTM layer enables the data to be processed in both forward and backward directions with the Forward Layer and the Backward Layer, respectively. The detailed description can be found in the literature [25].

Beyond the challenge of considering forward and backward information, another important issue is capturing the massive amount of uncertainty in the model and the baseline heat

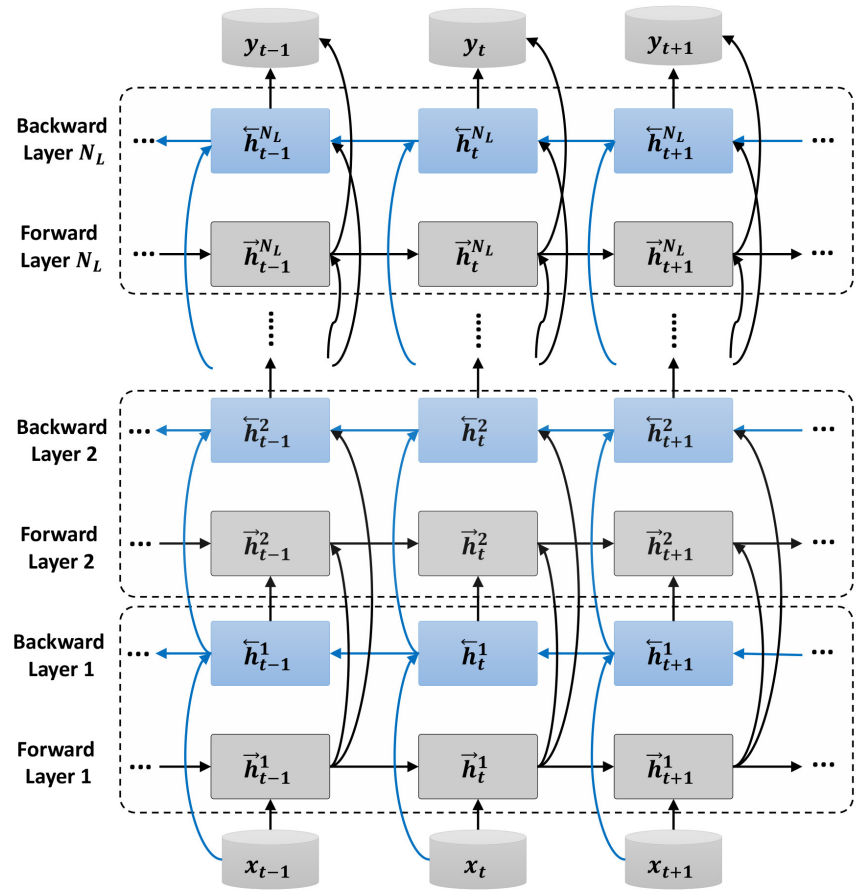

Fig. 2. Unfolded topological graph of a deep bidirectional LSTM network.

demand at the household level. In other words, "thinking" is one of the core, fundamental abilities for deep learning to build a real artificial intelligence system. Regarding the tasks of forecasting or baseline estimation, convectional deep recurrent neural networks (e.g., Bi-LSTM) can only provide a single point forecast or estimate for each time step whereas uncertainty cannot be represented and learned in this type of deterministic model. To this end, probabilistic graphical models (PGM) [26], which employ diagrammatic representations to depict random variables and relationships among them, enable deep LSTM networks to be capable of "thinking" about how certain the model is regarding its output estimates. In particular, this can be achieved by combining PGM and deep LSTM networks within a principled probabilistic framework called Bayesian deep LSTM networks, whose individual component (i.e., PGM and deep LSTM networks) can benefit from the synergy between them. Specifically, the primary advantages of Bayesian deep Bi-LSTM networks can be summarized as follows:

1) Bayesian deep Bi-LSTM networks (BDBL) provide a principled way of integrating PGM and deep Bi-LSTM networks to jointly capture the model uncertainty and the stochastic uncertainty for each time step while considering both the forward and backward dependencies.

2) Compared to conventional deterministic deep learning models, BDBL is inherently a probabilistic model whose parameters are expressed as conditional probability distributions. Therefore, the probabilistic estimates generated from the constructed BDBL model can be obtained by directly sampling its parameters instead of adding noise to the point estimate or setting up multiple input scenarios. 
3) The implicit regularization built in BDBL can deal with the overfitting problem, especially when the data are insufficient [21].

Given the training dataset $X=\left[x_{1}, \cdots, x_{N}\right]^{T} \in \mathbb{R}^{N \times M_{X}}$ and the training target output $Y=\left[y_{1}, \cdots, y_{N}\right]^{T} \in \mathbb{R}^{N \times M_{y}}$, the primary objective of training a BDBL model $f^{W}$ is to optimize its parameters $W$ by minimizing the estimation error between the actual baseline heat demand $y$ and the estimate $\hat{y}=f^{W}(X)$. In general, PGMs can be classified into directed PGMs and undirected PGMs and in this work, directed PGMs (i.e., Bayesian networks) are considered to be integrated with the deep Bi-LSTM networks. First, to capture the uncertainties in the model parameter and the model structure, a prior distribution $\mathcal{N}(0, I)$ is placed over the weights and bias. Then the likelihood of the model can be represented by a Gaussian distribution $p\left(y \mid f^{W}(x)\right)=N\left(f^{W}(x), \sigma^{2}\right)$ where $\sigma$ is noise level. Based on the training datasets, the posterior distribution $p(W \mid X, Y)$ can be obtained via Bayes rule, as follows:

$$
p(W \mid X, Y)=\frac{p(Y \mid X, W) \cdot p(W)}{p(Y \mid X)}
$$

where $p(Y \mid X)$ is the marginal probability that cannot be estimated analytically; however, it can be approximated via various inference techniques. As one of the most widely used inference approaches, variational inference (VI) is employed in this case to estimate the posterior $p(W \mid X, Y)$ by identifying an approximating variational distribution $q_{\theta}(W)$, parameterized by $\theta$ which minimizes the Kullback-Leibler (KL) divergence between $q_{\theta}(W)$ and $p(W \mid X, Y)$. In this way, the model uncertainty can be represented by the approximated predictive variance $\operatorname{var}(y) \approx E U\left(x, y, \hat{W}_{t}\right)+\sigma^{2}$ where

$$
E U\left(x, y, \hat{W}_{t}\right)=\frac{1}{T_{s}} \sum_{t=1}^{T_{s}} f^{\hat{W}_{t}}(x)^{T} f^{\hat{W}_{t}}(x)-E(y)^{T} E(y),
$$

$T_{s}$ is the number of sampled weights $\hat{W}_{t}$ that follows the dropout distribution $q_{\theta}(W)$. In addition, the approximated predictive mean is denoted by $E(y) \approx \sum_{t=1}^{T_{s}} f^{\hat{W}_{t}}(x) / T_{s}$. The term $E U\left(x, y, \hat{W}_{t}\right)$ indicates how uncertain the model is regarding its output, which measures the model uncertainty. It is notable that the model uncertainty can actually be diminished when there is sufficient training data.

For the inherently stochastic uncertainty, the loss function of the data dependent heteroscedastic model is given as follows:

$$
\mathcal{L}(\theta)=\frac{1}{2 T} \sum_{i=1}^{T} \frac{1}{\sigma\left(x_{i}\right)^{2}}\left\|y_{i}-f^{W}\left(x_{i}\right)\right\|^{2}+\frac{1}{2} \log \sigma\left(x_{i}\right)^{2}
$$

where $\theta$ is estimated via maximum a posteriori (MAP) inference method. To consider both the model and stochastic uncertainties in a single model, placing a distribution over the weights and bias of the heteroscedastic model can finally construct the proposed BDBL model. First, a new expression: $\left[\hat{y}, \hat{\sigma}^{2}\right]=f_{B D B L}^{\hat{W}}(x)$ is set up to include both the predictive mean and the predictive variance; Then, we need to determine the likelihood to model the stochastic uncertainty, and the loss function of the BDBL model can be expressed as: $\mathcal{L}_{B D B L}(\theta)=\frac{1}{T} \sum_{i=1}^{T} \frac{1}{2 \hat{\sigma}_{i}^{2}}\left\|y_{i}-\hat{y}_{i}\right\|^{2}+\frac{1}{2} \log \hat{\sigma}_{i}^{2}$. Furthermore, the final predictive uncertainty can be approximated to include both the model and the stochastic uncertainties, as follows:

$$
\operatorname{var}(y) \approx\left[\frac{1}{T} \sum_{t=1}^{T} \hat{y}_{t}{ }^{2}-\left(\frac{1}{T} \sum_{t=1}^{T} \hat{y}_{t}\right)^{2}\right]+\frac{1}{T} \sum_{t=1}^{T} \hat{\sigma}_{t}^{2} .
$$

Detailed illustrations and formulations of Bayesian deep learning can be found in the references [18], [20], and [21].

\section{CASE Study}

\section{A. Data Descriptions}

The Flexible Residential Energy Efficiency Demand Optimization and Management (FREEDOM) project is a trial of smart control technology for hybrid heat pumps that aims to explore advanced strategies for exploiting demand flexibility to create new value propositions and manage peak load [4]. The FREEDOM datasets used in this case consist of 10,176 half-hourly measurements of external temperature, tariff data, customer's highest room temperature set point, heat output from the gas boiler and from the heat pump, across 60 customers (i.e., after data cleaning) from 1st Oct 2017 to 30th Apr 2018. Regarding the interventions in the project, the E7 tariff is set for 12 customers during the period of 27-30th Nov 2017. In addition, the E10 tariff is set for other 12 customers during the period of 1-4th Dec 2017. All the other customers are considered as the non-event customers with a flat tariff.

\section{B. Experiment Setup}

To demonstrate the superiority of the proposed scheme, a number of state-of-the-art baseline estimation methods are implemented for comparison. Note that there is very limited work on the topic of baseline heat demand estimation; we consider the methods from the baseline electricity demand estimation literature: M1) High5of10 [9]; M2) Low5of10 [8]; M3) regression-based method [9]; M4) clustering-based method [10]; M5) Gaussian process (GP) [12]; M6) gradient boosting quantile regression (GBQR) [27]; M7) quantile random forests [27]; and M8) the proposed BDBL method. Regarding the evaluation metrics, the mean square error (MSE) and the mean absolute error (MAE) are employed to assess the point estimation performance. Furthermore, two comprehensive metrics, the pinball Loss function and the Winkler score are employed to evaluate the probabilistic estimation performance regarding the main factors of calibration, sharpness and reliability. Detailed formulations of these metrics can be found in [28] and [29]. Additionally, the hyperparameters of the proposed BDBL model determined via grid search and cross validation are given in Table I. In this work, the algorithm was implemented in Python with the main packages of Keras [30] and Edward [31] and run on an Intel Xeon PC with NIVIDIA Titan-V GPU.

\section{Baseline Estimation Results}

To compare the estimation performance between the proposed BDBL method and other state-of-the-art approaches, the deterministic estimation results of $\operatorname{MSE}\left(\mathrm{kW}^{2}\right)$ and $\operatorname{MAE}(\mathrm{kW})$ 
TABLE I

PARAMETER SETTING FOR BDBL

\begin{tabular}{ll}
\hline Parameter & Value \\
\hline Number of layers & 8 \\
Number of neurons per layer & 10 \\
Batch size & 192 \\
Maximum number of epochs & 100 \\
Optimizer & Adam \\
Learning rate & 0.001 \\
Number of samples & 150 \\
Prior on parameters & Standard Normal Distribution \\
\hline
\end{tabular}

and the probabilistic estimation results of the pinball loss $(\mathrm{kW})$ and the Winkler Score $(\mathrm{kW})$ are presented in Table II and Table III, respectively. Note that, as employed in [6], the experiments are conducted on 36 non-event customers and 5 test customers selected from the non-event group to quantitatively evaluate the performance of the baseline estimation methods.

TABLE II

Deterministic BASELINE Estimation PERFoRMANCE

\begin{tabular}{lcc}
\cline { 2 - 3 } & MSE(kW2 $)$ & MAE(kW) \\
\hline M1:High5of10 & 6.3802 & 1.4254 \\
M2:Low5of10 & 6.6002 & 1.4186 \\
M3:Regression & 2.9019 & 1.1367 \\
M4:Clustering & 6.6033 & 1.4182 \\
M5:GP & 6.8491 & 1.5653 \\
M6:GBQR & 3.3452 & 1.1184 \\
M7:QRF & 3.1624 & 1.0776 \\
M8:BDBL & 1.2852 & 0.7693 \\
\hline
\end{tabular}

TABLE III

Probabilistic Baseline Estimation Performance

\begin{tabular}{lcc} 
& Pinball Loss $(\mathrm{kW})$ & Winkler Score $(\mathrm{kW})$ \\
\hline M5:GP & 1.1185 & 26.3541 \\
M6:GBQR & 0.4092 & 6.5119 \\
M7:QRF & 0.3762 & 5.1926 \\
M8:BDBL & 0.2703 & 3.5869 \\
\hline
\end{tabular}

The tables show that the proposed BDBL model M8 outperforms other conventional methods with respect to both deterministic and probabilistic estimation results, indicated by lower evaluation metric values. More specifically, compared with the best performing deterministic estimation method (i.e., M3), approximately $55.71 \%$ and $32.32 \%$ improvements are obtained by using the proposed M8 regarding the metric values of MSE and MAE, respectively. Furthermore, the remarkable capability of capturing the uncertainty of residential customers can be demonstrated based on the probabilistic estimation results, as indicated by approximately $28.14 \%$ lower pinball loss and $30.92 \%$ lower Winkler score values when compared with the second-best probabilistic approach, QRF (M7). Additionally, Fig. 3 shows the estimation results of the four test days with E10 events for one customer obtained via the proposed BDBL model (M7). Note that the actual baseline heat demand is represented by the yellow curve and the $5^{t h}, 25^{t h}, 50^{t h}$, $75^{\text {th }}$ and $95^{\text {th }}$ quantiles are presented in. It can be seen that, although the actual baseline heat demand at household level exhibits high variability and low predictability, the proposed BDBL model can still generate accurate probabilistic estimates

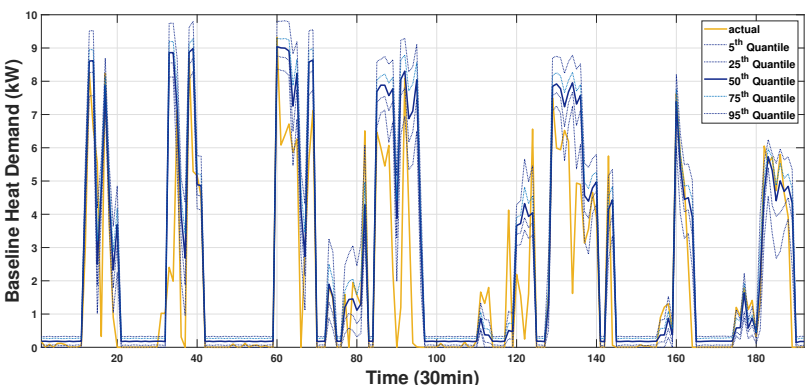

Fig. 3. Estimated baseline heat demand of one customer for E10 event days.

with a tight converge interval and a low predicted interval varying over time.

\section{Quantifying SHHP Flexibility}

After demonstrating the superior performance of the proposed baseline estimation method, in this section, we employ the BDBL-based flexibility quantification method to estimate how much of the heat pump demand has been shifted under E7 and E10 events compared to the baseline heat demand based on the flat electricity tariff.

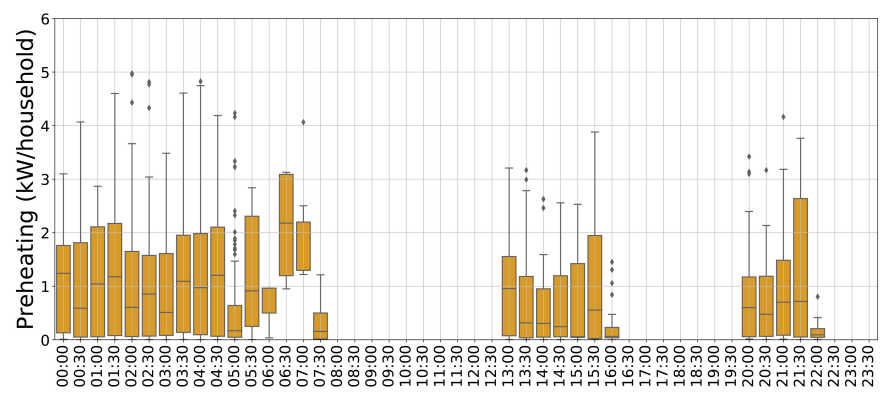

Fig. 4. Quantified preheating for all customers during E7 and E10 events.

Fig. 4 presents the boxplots of the quantified volume of heat used for preheating across all customers during the E7 and E10 events. Note that the central mark indicates the median value and the upper and lower bounds represent the maximum and minimum preheating values observed in the experiments, respectively. Note that each boxplot contains the samples of quantified preheating for all 24 customers (i.e., 150 samples per customer). The preheating half-hour periods for a given customer were detected based on the customers room temperature set point, so that the preheating intervals only included those half-hour periods in the low-price windows that had lower temperature set points compared to the highest set point value over a day. As can be seen, the smart hybrid heat pumps can provide a considerable amount of flexibility, quantified by the preheating during the event periods, especially for the midnight hours. This is because the external temperature and the customer's highest temperature set point during the midnight hours from 0:30 to 7:30 are usually lower than those of the afternoon and evening hours (i.e., 13:00-16:00 and 20:00-22:00). 


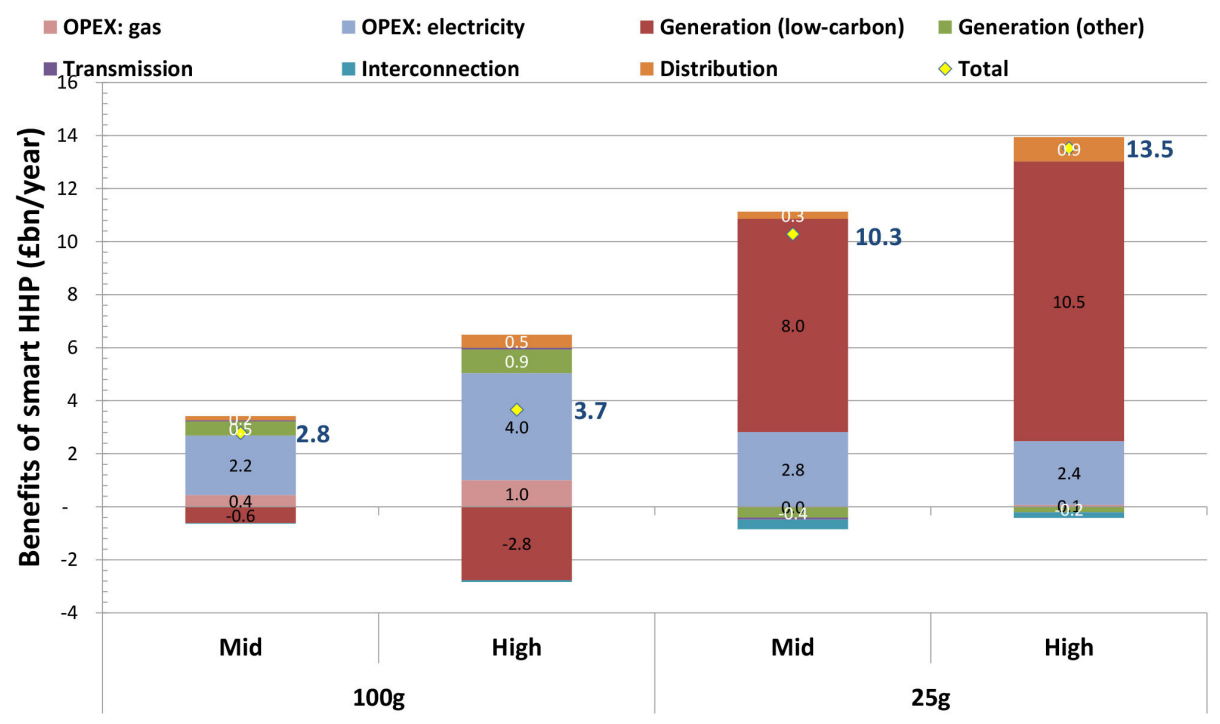

Fig. 5. Benefits of rolling-out SHHPs to the future GB electricity systems.

\section{E. Benefits to The Future GB Electricity System}

In this study, the benefits of the SHHPs (through "preheating") have been investigated and quantified by inputting the volume of heat pump output shifted as the result of varying price experiments, compared to the baseline profile, into the whole-system modeling where the capability of shifting HP output in time has been built into the Whole-electricity System Investment Model (WeSIM), that minimizes the total operation and investment cost of the power system subject to security and carbon constraints. More specifically, this holistic model identifies optimal decisions for investing in generation, network and/or storage capacity, in order to satisfy the realtime supply-demand balance in an economically optimal way, while at the same time ensuring efficient levels of security of supply. The WeSIM has been extensively tested in previous research studying the interconnected electricity systems of the UK and the rest of Europe. An advantage of WeSIM over most traditional models is that it is able to simultaneously consider system operation decisions and capacity additions to the system, with the ability to quantify trade-offs of using alternative mitigation measures, such as DSR and storage, for real-time balancing and transmission and distribution network and/or generation reinforcement management. More details regarding the whole-system model are provided in [32]. Two levels of flexibility have been investigated: (i) medium and (ii) highly flexible HHP cases corresponding to $25 \%$ and $100 \%$ of the households that participated in the providing flexibility through smart control of HHPs.

The results of the studies are presented in Fig. 5, which shows the difference between the system costs of the HHPs without and with smart preheating capability for the 100 and $25 \mathrm{gCO}_{2} / \mathrm{kWh}$ carbon targets. The CapEx and the OPEX represent the capital expenditure and the operating expenses, respectively. It is important to note that in this study, it was assumed that there was no other demand flexibility or new electricity storage in the system. Therefore, improving the system flexibility through smart control of HHPs will create substantial benefits, and the smart control will have high value. In addition, the results demonstrate the following:

i) In the $100 \mathrm{gCO}_{2} / \mathrm{kWh}$ cases, the smart control of HHPs increases the cost of low-carbon generation CapEx, but the investment in low-carbon generation also reduces the operating cost of electricity. As nuclear and renewables are zeromarginal-cost plants, increased capacity of these technologies reduces the operating cost. The increased investment in lowcarbon generation reduces the utilization of gas.

ii) In the $25 \mathrm{gCO}_{2} / \mathrm{kWh}$ cases, the flexibility provided by the smart control of HHPs enhances utilization of renewables and other low-carbon technologies. This reduces the capacity of, and corresponding investment in, low-carbon generation.

iii) The smart control of HHPs also contributes to savings in distribution network reinforcement, which is not unexpected, as preheating can further reduce the peak demand.

iv) The value of the smart control of HHPs increases from $2.8-3.7 \mathrm{bn} / \mathrm{year}$ in the $100 \mathrm{gCO} 2 / \mathrm{kWh}$ cases to $10.3-13.5$ bn/year in the $25 \mathrm{gCO}_{2} / \mathrm{kWh}$ cases.

\section{Conclusions}

In this paper, a novel data-driven probabilistic preheating quantification approach is proposed to estimate the flexible heating of fully optimized hybrid heat pumps. First, to address the challenge of capturing the model uncertainty and the stochastic uncertainty, BDBL is developed to enable deep learning to represent these uncertainties via combining PGM with deep Bi-LSTM networks. Additionally, the novel feature design procedure and the bidirectional LSTM architecture can fully utilize the pre-event and post-event information to enhance the estimation accuracy. Case studies are conducted based on the data collected from the world's first sizable field trial of smart hybrid heat pumps. The superior estimation performance of the proposed scheme is demonstrated regarding the deterministic and probabilistic estimation results. Finally, the quantified flexibility is used as an input into the wholesystem model to quantify the benefits of rolling out the SHHPs 
to future GB electricity systems. The results show that the value of SHHPs increases from $2.8-3.7 \mathrm{bn} /$ year in the 100 $\mathrm{gCO}_{2} / \mathrm{kWh}$ cases to $10.3-13.5 \mathrm{bn} /$ year in the $25 \mathrm{gCO}_{2} / \mathrm{kWh}$ cases.

Future work will focus on employing more advanced Bayesian deep learning approach such as directly estimating the prior distribution from the data to improve the quantification accuracy. Furthermore, we can build a time-varying quantification model to include different nonDR customer features for the examined DR customer during different periods (e.g., seasons). Additionally, the quantified preheating can be used to assess the impact of SHHPs on electricity and gas distribution networks.

\section{REFERENCES}

[1] X. Zhang, G. Strbac, F. Teng, and P. Djapic, "Economic assessment of alternative heat decarbonisation strategies through coordinated operation with electricity system-uk case study," Applied Energy, vol. 222, pp. 79-91, Jul. 2018.

[2] S. Heinen, D. Burke, and M. O'Malley, "Electricity, gas, heat integration via residential hybrid heating technologies - an investment model assessment," Energy, vol. 109, pp. 906 - 919, Aug. 2016.

[3] Wales\&WestUtilities, "Freedom project: Final report," Freedom Project, Tech. Rep., 2018.

[4] E. Carter, O. Lancaster, and F. Chanda, "Early results from the freedom project: fully-optimised hybrid heat pumps providing demand flexibility," in 12th IEA heat pump conference 2017: Rotterdam, 2017.

[5] C. Calder, "Taking a hybrid approach to decarbonise domestic heating," Energy World, pp. 30-31, Jun. 2018.

[6] F. Wang, K. Li, C. Liu, Z. Mi, M. Shafie-Khah, and J. P. S. Catalo, "Synchronous pattern matching principle-based residential demand response baseline estimation: Mechanism analysis and approach description," IEEE Transactions on Smart Grid, vol. 9, no. 6, pp. 6972-6985, Nov. 2018.

[7] Y. Wang, Q. Chen, T. Hong, and C. Kang, "Review of smart meter data analytics: Applications, methodologies, and challenges," IEEE Transactions on Smart Grid, in press.

[8] T. K. Wijaya, M. Vasirani, and K. Aberer, "When bias matters: An economic assessment of demand response baselines for residential customers," IEEE Transactions on Smart Grid, vol. 5, no. 4, pp. 17551763, Jul. 2014

[9] S. Mohajeryami, M. Doostan, A. Asadinejad, and P. Schwarz, "Error analysis of customer baseline load (cbl) calculation methods for residential customers," IEEE Transactions on Industry Applications, vol. 53, no. 1, pp. 5-14, Jan.-Feb. 2017.

[10] Y. Zhang, W. Chen, R. Xu, and J. Black, "A cluster-based method for calculating baselines for residential loads," IEEE Transactions on Smart Grid, vol. 7, no. 5, pp. 2368-2377, Sept. 2016.

[11] L. Hatton, P. Charpentier, and E. Matzner-Løber, "Statistical estimation of the residential baseline," IEEE Transactions on Power Systems, vol. 31, no. 3, pp. 1752-1759, May 2016.

[12] Y. Weng, J. Yu, and R. Rajagopal, "Probabilistic baseline estimation based on load patterns for better residential customer rewards," International Journal of Electrical Power \& Energy Systems, vol. 100, pp. 508-516, Sept. 2018.

[13] M. Valls, A. Bello, J. Reneses, and P. Fras, "Probabilistic characterization of electricity consumer responsiveness to economic incentives," Applied Energy, vol. 216, pp. 296 - 310, Apr. 2018.

[14] H. Shi, M. Xu, and R. Li, "Deep learning for household load forecastinga novel pooling deep rnn,” IEEE Transactions on Smart Grid, vol. 9, no. 5, pp. 5271-5280, Sep. 2018.

[15] W. Kong, Z. Y. Dong, Y. Jia, D. J. Hill, Y. Xu, and Y. Zhang, "Short-term residential load forecasting based on 1stm recurrent neural network," IEEE Transactions on Smart Grid, vol. 10, no. 1, pp. 841-851, Jan. 2019.

[16] M. Khodayar, O. Kaynak, and M. E. Khodayar, "Rough deep neural architecture for short-term wind speed forecasting," IEEE Transactions on Industrial Informatics, vol. 13, pp. 2770-2779, Dec. 2017.

[17] X. Luo, J. Sun, L. Wang, W. Wang, W. Zhao, J. Wu, J. Wang, and Z. Zhang, "Short-term wind speed forecasting via stacked extreme learning machine with generalized correntropy," IEEE Transactions on Industrial Informatics, vol. 14, no. 11, pp. 4963-4971, Nov. 2018.
[18] Y. Gal, "Uncertainty in deep learning," Ph.D. dissertation, University of Cambridge, 2016

[19] R. G. Junker, A. G. Azar, R. A. Lopes, K. B. Lindberg, G. Reynders, R. Relan, and H. Madsen, "Characterizing the energy flexibility of buildings and districts," Applied Energy, vol. 225, pp. 175-182, Sept. 2018.

[20] A. Kendall and Y. Gal, "What uncertainties do we need in bayesian deep learning for computer vision?" in Advances in Neural Information Processing Systems 30, I. Guyon, U. V. Luxburg, S. Bengio, H. Wallach, R. Fergus, S. Vishwanathan, and R. Garnett, Eds. Curran Associates, Inc., 2017, pp. 5574-5584.

[21] H. Wang and D.-Y. Yeung, "Towards bayesian deep learning: A framework and some existing methods," IEEE Transactions on Knowledge and Data Engineering, vol. 28, no. 12, pp. 3395-3408, Dec. 2016.

[22] M. Sun, I. Konstantelos, and G. Strbac, "A deep learning-based feature extraction framework for system security assessment," IEEE Transactions on Smart Grid, in press.

[23] S. Hochreiter, Y. Bengio, P. Frasconi, and J. Schmidhuber, "Gradient flow in recurrent nets: the difficulty of learning long-term dependencies," 2001.

[24] S. Hochreiter and J. Schmidhuber, "Long short-term memory," Neural computation, vol. 9, no. 8, pp. 1735-1780, Dec. 1997.

[25] A. Graves, S. Fernández, and J. Schmidhuber, "Bidirectional 1stm networks for improved phoneme classification and recognition," in Artificial Neural Networks: Formal Models and Their Applications - ICANN 2005. Berlin, Heidelberg: Springer Berlin Heidelberg, 2005, pp. 799-804.

[26] C. M. Bishop, Pattern Recognition and Machine Learning (Information Science and Statistics). Berlin, Heidelberg: Springer-Verlag, 2006

[27] Y. Wang, N. Zhang, Y. Tan, T. Hong, D. S. Kirschen, and C. Kang, "Combining probabilistic load forecasts," IEEE Transactions on Smart Grid, in press.

[28] T. Hong and S. Fan, "Probabilistic electric load forecasting: A tutorial review," International Journal of Forecasting, vol. 32, no. 3, pp. 914 938, Jul.-Sept. 2016.

[29] M. Sun, Y. Wang, G. Strbac, and C. Kang, "Probabilistic peak load estimation in smart cities using smart meter data," IEEE Transactions on Industrial Electronics, vol. 66, no. 2, pp. 1608-1618, Feb. 2019.

[30] F. Chollet et al., "Keras," https://keras.io, 2015.

[31] D. Tran, A. Kucukelbir, A. B. Dieng, M. Rudolph, D. Liang, and D. M. Blei, "Edward: A library for probabilistic modeling, inference, and criticism," arXiv preprint arXiv:1610.09787, 2016.

[32] D. Pudjianto, M. Aunedi, P. Djapic, and G. Strbac, "Whole-systems assessment of the value of energy storage in low-carbon electricity systems," IEEE Transactions on Smart Grid, vol. 5, no. 2, pp. 10981109, Mar. 2014.

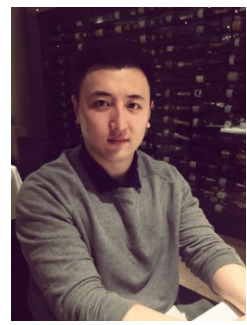

Mingyang Sun (M'16) received the Ph.D. degree in Electrical and Electronic Engineering from Imperial College London, U.K., in 2017.

$\mathrm{He}$ is currently a Research Associate at Imperial College London. His current research interests include big data analytics and artificial intelligence in energy systems.

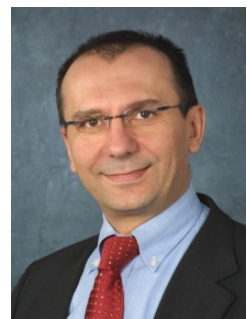

Goran Strbac (M'95) is a Professor of electrical energy systems at Imperial College, London, U.K. His current research interests include electricity generation, transmission and distribution operation, planning and pricing, and integration of renewable and distributed generation in electricity systems. 


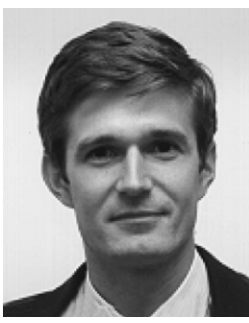

Predrag Djapic (M03) M.Sc. degree in electrical engineering from University of Belgrade, Belgrade, Serbia, in 1999. He is currently a Researcher with the Imperial College London, London, U.K. He has more than 20 years of international experience in electricity system modeling, planning, and analysis.

His work includes development of models and evaluation of distribution network load related expenditure driven by the uptake of distributed generation and low carbon technologies, and contribution to review and update of GB electricity distribution, and transmission systems security of supply standards.

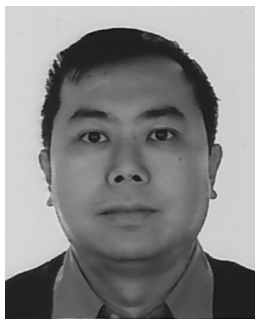

Danny Pudjianto (M98) received the B.Sc. degree from the Institut Teknologi Surabaya, Indonesia, in 1996, and the M.S. and Ph.D. degrees from the University of Manchester Institute of Science and Technology, Manchester, U.K., in 1999 and 2003, respectively. Currently, he is a Research Fellow with Imperial College London, London, U.K. 